\title{
Social Status and Sex Effects on Neural Morphology in Damaraland Mole-Rats, Fukomys damarensis
}

\author{
Jeff J. Anyan ${ }^{a}$ Marianne L. Seney ${ }^{b}$ Amanda Holley $^{b}$ Lynn Bengston $^{b}$ \\ Bruce D. Goldman ${ }^{c}$ Nancy G. Forger ${ }^{b}$ Melissa M. Holmes ${ }^{a}$ \\ aDepartment of Psychology, University of Toronto Mississauga, Mississauga, Ont., Canada; \\ ${ }^{b}$ Department of Psychology and Center for Neuroendocrine Studies, University of Massachusetts, Amherst, Mass., \\ and ${ }^{\mathrm{C}}$ Department of Ecology and Evolutionary Biology, University of Connecticut, Storrs, Conn., USA
}

\begin{abstract}
Key Words
Bed nucleus of the stria terminalis - Damaraland molerat $\cdot$ Medial amygdala $\cdot$ Naked mole-rat $\cdot$ Onuf's nucleus • Paraventricular nucleus $\cdot$ Sex difference $\cdot$ Social status
\end{abstract}

\begin{abstract}
We previously reported that in a eusocial rodent, the naked mole-rat (Heterocephalus glaber), traditional neural sex differences were absent; instead, neural dimorphisms were associated with breeding status. Here we examined the same neural regions previously studied in naked mole-rats in a second eusocial species, the Damaraland mole-rat (Fukomys damarensis). Damaraland mole-rats live in social groups with breeding restricted to a small number of animals. However, colony sizes are much smaller in Damaraland mole-rats than in naked mole-rats and there is consequently less reproductive skew. In this sense, Damaraland mole-rats may be considered intermediate in social organization between naked mole-rats and more traditional laboratory rodents. We report that, as in naked mole-rats, breeding Damaraland molerats have larger volumes of the principal nucleus of the bed nucleus of the stria terminalis and paraventricular nucleus of the hypothalamus than do subordinates, with no effect of sex on these measures. Thus, these structures may play spe-
\end{abstract}

cial roles in breeders of eusocial species. However, in contrast to what was seen in naked mole-rats, we also found sex differences in Damaraland mole-rats: volume of the medial amygdala and motoneuron number in Onuf's nucleus were both greater in males than in females, with no significant effect of breeding status. Thus, both sex and breeding status influence neural morphology in Damaraland mole-rats. These findings are in accord with the observed sex differences in body weight and genitalia in Damaraland but not naked mole-rats. We hypothesize that the increased sexual dimorphism in Damaraland mole-rats relative to naked mole-rats is related to reduced reproductive skew.

Copyright $\odot 2011$ S. Karger AG, Basel

\section{Introduction}

Sexual dimorphism is a common feature of the mammalian nervous system, with sex differences in structure and/or function identified in most species studied to date. These dimorphisms are thought to relate to sex differences in reproductive physiology and behavior and presumably evolved as specializations that support the different reproductive strategies of males and females. The vast majority of what we know about sex differences

\section{KARGER}

() 2011 S. Karger AG, Basel

Fax +4161306 1234

E-Mail karger@karger.ch

www.karger.com
Accessible online at: www.karger.com/bbe
Melissa M. Holmes, $\mathrm{PhD}$

Department of Psychology, University of Toronto Mississauga

3359 Mississauga Road North

Mississauga, ON L5L 1C6 (Canada)

Tel. +1 905828 3956,E-Mail melissa.holmes@utoronto.ca 
in the nervous system comes from species in which adult males and females have distinct reproductive roles and associated sex-typical behavioral profiles. More recently, we have begun to explore how social variables might influence sex differences in the mammalian nervous system. Specifically, we wondered if neural sexual dimorphisms are reduced or absent in species in which most individuals do not become reproductive. Eusocial mammals that exhibit striking social and reproductive hierarchies provide an elegant natural experiment with which to ask these questions.

We recently reported the near absence of neural sex differences in the eusocial naked mole-rat (Heterocephalus glaber). These animals live in large fossorial colonies, typically including 60-80 individuals, in which reproduction is restricted to a single breeding female, i.e. the queen, and 1-3 breeding males [Jarvis, 1981]. The other animals in the colony, called subordinates, are reproductively suppressed and obtain inclusive fitness by supporting the reproductive efforts of the breeders. Subordinate naked mole-rats are not sexually dimorphic with regard to body size or the morphology of their external genitalia [Lacey and Sherman, 1991; Peroulakis et al., 2002; Seney et al., 2009] and do not mate or show sex differences in other behaviors [Jarvis, 1991; Lacey et al., 1991; Lacey and Sherman, 1991].

We compared regional volume, cell number, and cell size in several brain regions linked to reproduction in subordinate and breeder naked mole-rats, focusing on areas that are sexually dimorphic in other rodents, and found no sex differences on any measure. However, breeders, regardless of sex, had larger volumes of the principal nucleus of the bed nucleus of the stria terminalis (BSTp), paraventricular nucleus (PVN), and medial amygdala (MeA) [Holmes et al., 2007]. We also examined the spinal motoneurons that innervate striated perineal muscles [referred to as the spinal nucleus of the bulbocavernosus or Onuf's nucleus (ON) depending on the species-specific organization of motoneurons], which are sexually dimorphic in every other species studied to date. We again found no sex differences in ON motoneurons in either subordinate or breeding naked mole-rats [Peroulakis et al., 2002; Seney et al., 2006], but breeders, regardless of sex, had more large motoneurons in ON than did subordinates [Seney et al., 2006]. Collectively, these data suggest that social status might play a more important role than sex in determining the morphology of the naked mole-rat nervous system.

Eusociality is also thought to occur in at least 1 other bathyergid species, the Damaraland mole-rat (Fukomys damarensis; formerly Cryptomys damarensis) [Bennett and Jarvis, 1988; Kock et al., 2006]. Damaraland molerats exhibit less reproductive skew than do naked molerats; each colony contains an average of about 16 animals with a single breeding pair, and more individuals are estimated to achieve direct reproductive fitness in Damaraland mole-rats than in naked mole-rats $(10$ and $0.1 \%$, respectively) [Jarvis and Bennett, 1993; Jarvis et al., 1994]. Interestingly, phylogenetic analysis and comparison of colony dynamics indicate that eusociality evolved independently in these 2 species [Allard and Honeycutt, 1992; Jarvis and Bennett, 1993]. This provides an excellent opportunity to investigate evolved associations between sociality, reproductive skew, and sex differences in the nervous system.

Thus, in the present report we compared the nervous systems of male and female breeding and subordinate Damaraland mole-rats, focusing exclusively on neural regions previously examined in naked mole-rats. Specifically, we asked whether the volume of the BSTp, PVN, and $\mathrm{MeA}$, or the number of large motoneurons in $\mathrm{ON}$ is affected by sex or breeding status in Damaraland molerats. Each of these features is sexually dimorphic in other rodents but differs by breeding status and not sex in naked mole-rats.

\section{Animals and Methods}

\section{Animals and Housing}

Damaraland mole-rats were maintained at the University of Connecticut, Storrs. Colonies were housed in polypropylene tubs connected by lengths of acrylic tubing. Each tub was lined with corncob bedding. Animals were fed ad libitum with a diet consisting of sweet potatoes, apples, squash, and oatmeal. Animal rooms were maintained on a 16:8 light:dark photoperiod, and room temperature was kept between 22 and $26^{\circ} \mathrm{C}$.

We compared breeder males $(n=5)$, breeder females $(n=6)$, subordinate males $(n=7)$, and subordinate females $(n=8)$. Breeders were generated by removing subordinate adult Damaraland mole-rats from their natal colonies and placing them with an opposite sex partner. These animals were paired for at least 2 years prior to tissue collection; all pairs had produced at least 1 litter although no pups were successfully raised by any pair. Animals assigned to the subordinate condition remained in their natal colony until tissue collection.

All procedures were approved by the Institutional Animal Care and Use Committee of the University of Connecticut (protocol No. A07-046) and adhered to institutional and NIH guidelines for animal care.

Tissue Collection and Processing

The processing of brains and spinal cords closely followed those methods used previously for studies on naked mole-rats 
Fig. 1. Photomicrographs demonstrating the location of nuclei in thionin-stained coronal sections of the Damaraland molerat brain $(\mathbf{a}-\mathbf{c})$ and a Klüver-Barrerastained coronal section of the spinal cord (d). Shown are the BSTp (a), PVN and SCN (b), MeA (c), and ON and RDLN (d). Scale bar shown in $\mathbf{d}=500 \mu \mathrm{m}(\mathbf{a}-\mathbf{c})$ and $300 \mu \mathrm{m}$ (d).

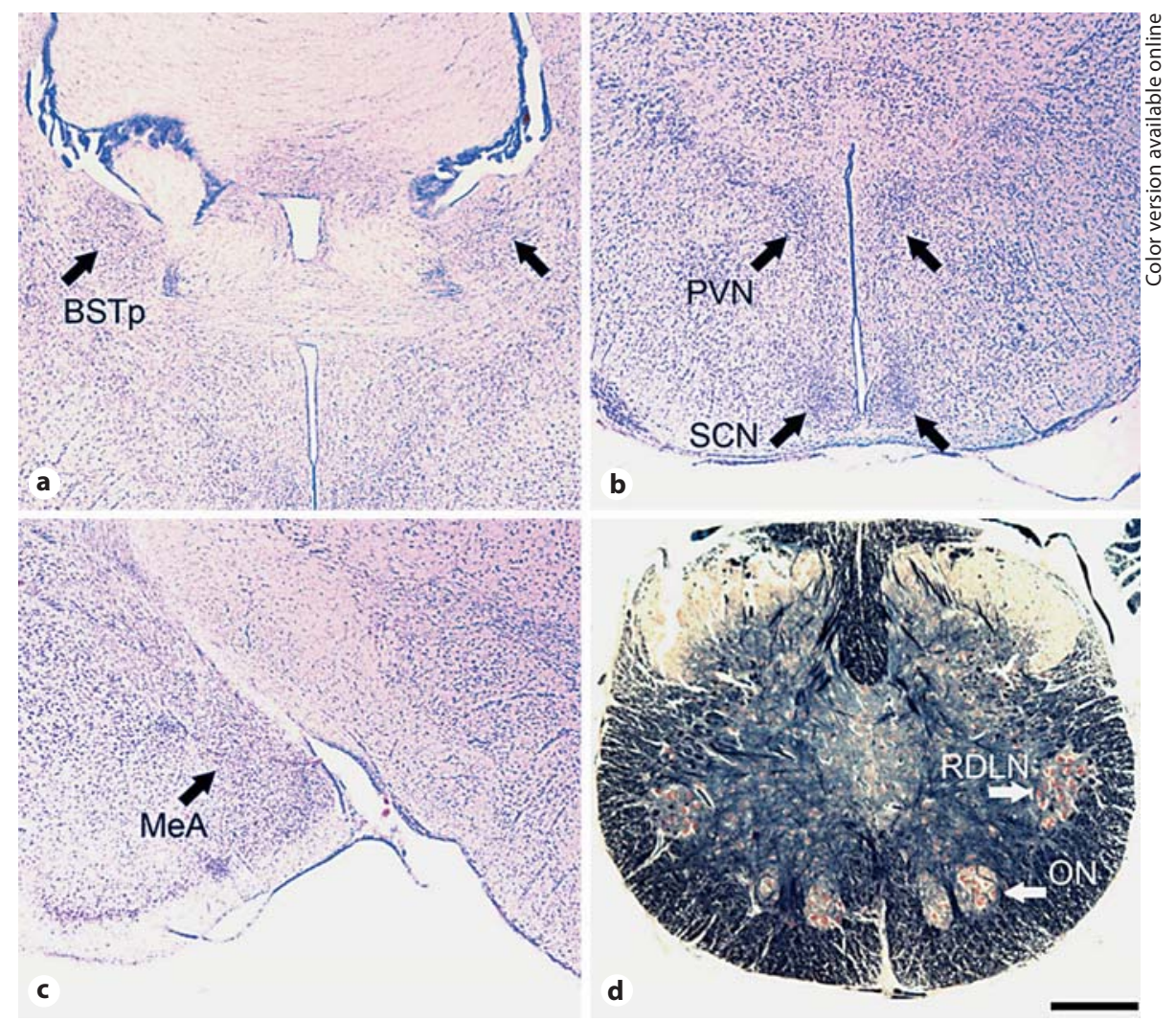

[Seney et al., 2006; Holmes et al., 2007]. Animals were weighed, overdosed with Avertin, and rapidly decapitated. Brains were removed and immersion-fixed in 5\% acrolein in phosphate buffer (PB) for $14 \mathrm{~h}$. Brains were then transferred to $30 \%$ sucrose in $\mathrm{PB}$ for approximately $72 \mathrm{~h}$, frozen-sectioned at $30 \mu \mathrm{m}$ in the coronal plane into 3 series, and stored in cryoprotectant (30\% sucrose, $30 \%$ ethylene glycol, and $1 \%$ PVP- 40 in $0.1 \mathrm{M} \mathrm{PBS}$ ) at $-20^{\circ} \mathrm{C}$ until use. Two of the 3 series were rinsed $3 \times 5$ min in PBS and mounted onto gel-coated slides. Slides were stained with thionin, dehydrated in an ascending series of alcohol, and coverslipped with Permount.

For analysis of ON, we first confirmed the location of motoneurons innervating the perineal muscles by injecting the retrograde tracer, cholera toxin-conjugated horseradish peroxidase, into the perineum of several animals as previously described [Peroulakis et al., 2002]. All labeled cells were located in a single motor pool that also stands out as a myelin-poor region surrounded by a halo of fibers in Klüver-Barrera-stained spinal cord sections (fig. 1d). For the analyses described here, vertebral columns were removed and immersion fixed in $10 \%$ formalin for approximately 10 days prior to transfer to Bouin's solution for 3 weeks. Spinal cords were then removed, embedded in paraffin, and sectioned in the coronal plane at $10 \mu \mathrm{m}$. Serial sections were mounted onto slides and stained with Klüver-Barrera.

\section{Tissue Analyses}

Regional brain volumes and cell size were acquired through the BSTp, PVN, and MeA (fig. la-c). Each of these regions showed increased volume (with no changes in cell size or cell number) in breeder naked mole-rats [Holmes et al., 2007]. We also examined the suprachiasmatic nucleus (SCN) as a control region (fig. 1b); the SCN was not expected to be sexually dimorphic and no effect of breeding status was seen in naked mole-rats [Holmes et al., 2007]. Volume estimates were calculated by tracing the cross-sectional area bilaterally through the rostrocaudal extent of each brain region using image analysis software (ImageJ, developed by the NIH and available as a free download). The total cross-sectional area was then multiplied by the section thickness $(30 \mu \mathrm{m})$ and sampling ratio (in this case, 1.5 because 2 out of 3 series of sections were analyzed). For the BSTp, tracings included the anteromedial and posteromedial subdivisions (corresponding to bregma 0.02 to $-0.22 \mathrm{~mm}$; plates $31-33$ in the mouse brain atlas of Franklin and Paxinos [2008]). For the PVN, tracings included the lateral and medial divisions of the magnocellular and parvocellular regions (corresponding to bregma -0.46 to $-1.22 \mathrm{~mm}$; plates 38-41 in the mouse brain atlas of Franklin and Paxinos [2008]). For the MeA, tracings included the posterodorsal and ventral subdivisions, which are not easily distinguishable in the mole-rat brain (corresponding to bregma -1.34 to $-2.30 \mathrm{~mm}$; plates $42-50$ in the mouse brain atlas of Franklin and Paxinos [2008]). For the SCN, tracings included all sections from bregma -0.34 to $-0.82 \mathrm{~mm}$ (plates $34-38$ in the mouse brain atlas of Franklin and Paxinos [2008]). Potential laterality effects were not addressed as we were unable to reliably distinguish left versus right hemispheres for all animals. For cell size measures, the cross-sectional area of at least 30 cells in each of 4 separate tissue 
Table 1. Mean age and body weight for male and female Damaraland mole-rats

\begin{tabular}{lll}
\hline & Age, months & Body weight, $g$ \\
\hline Breeders & & \\
$\quad$ Males $(\mathrm{n}=5)$ & $76.2(7.8)^{\mathrm{a}}$ & $201.7(11.0)^{\mathrm{b}}$ \\
$\quad$ Females $(\mathrm{n}=6)$ & $76.7(5.7)^{\mathrm{a}}$ & $151.3(12.2)$ \\
Subordinates & & \\
$\quad$ Males $(\mathrm{n}=7)$ & $33.9(5.8)$ & $178.5(20.6)^{\mathrm{b}}$ \\
$\quad$ Females $(\mathrm{n}=8)$ & $31.9(4.5)$ & $144.4(12.1)$ \\
\hline
\end{tabular}

Values are presented as means (SEM).

a Significant main effect of social status.

${ }^{\mathrm{b}}$ Significant main effect of sex. sections, for a total of at least 120 cells, were traced and averaged within each region of every animal.

For analysis of $\mathrm{ON}$, all cells that contained visible nucleoli and that fell within the 'halo' in Klüver-Barrera-stained sections were counted in alternate sections through the extent of the nucleus for every animal. All cells in $\mathrm{ON}$ met the normal criteria for motoneurons; we did not find a population of 'small cells' as previously described in ON of naked mole-rats [Seney et al., 2006]. The mean cell size was determined by tracing at least 40 cells from each animal. As a control, cell counts and cell size analysis were also performed for the retrodorsolateral nucleus (RDLN), a motor pool in the lateral motor column at the same level as ON. In other rodents, RDLN motoneurons innervate muscles of the hind foot.

All dependent measures were analyzed using 2-way ANOVAs (sex by status). In addition, regression analyses were performed to evaluate possible relationships between age and neural morphology. Because breeders were significantly older than subordinates (see below), regressions were performed separately for breeders and subordinates but included both males and females within each reproductive class.

\section{Results}

\section{Age and Body Weight}

Damaraland mole-rats reach adult body size by approximately 14 months of age [Bennett et al., 1991]. They are among the longest-lived rodents, surviving over 15 years in captivity [Sura et al., 2010], and breeders remain reproductively active throughout their long lifespan. Table 1 provides the mean ages and body weights of all animals used in this study $(\mathrm{n}=26)$. The animals in the current study ranged between 1.8 and 8.5 years of age. Thus, all were in young-to-mid adulthood. However, the breeders available for this study were older than the subordinates $\left(\mathrm{F}_{1,22}=55.68 ; \mathrm{p}<0.001\right)$. Age did not vary by sex and there was no sex-by-status interaction. Consistent with previous reports, we observed a sex difference in body weight; males were heavier than females $\left(\mathrm{F}_{1,22}=\right.$ $7.53 ; \mathrm{p}=0.01)$ with no effect of status and no sex-by-status interaction.

Not all animals were used in all analyses. A total of 20 animals were used for brain measures and an overlapping but not identical cohort of 19 animals was available for spinal cord analyses. When analyzed separately for the brain and spinal cord cohorts, the main effect of age (breeders older than subordinates) remained significant in both cases. The main effect of sex on body weight that was seen when all animals were considered (male $>$ female) remained significant for the subset of animals used for brain analyses but not for the subset used for spinal cord analyses.

\section{Regional Brain Volumes and Cell Size}

As in naked mole-rats [Holmes et al., 2007], neither breeding nor subordinate Damaraland mole-rats of either sex have a distinct, darkly stained group of cells in the preoptic area that might be equivalent to the sexually dimorphic nucleus of the preoptic area [Gorski et al., 1978] found in rats.

BSTp volume was significantly larger in breeders than in subordinates (main effect of status: $\mathrm{F}_{1,16}=19.17$; $\mathrm{p}<$ 0.001 ; fig. 2a) with no main effect of sex. A similar pattern was seen in the PVN, with larger volumes in breeders than in subordinates $\left(\mathrm{F}_{1,16}=13.02 ; \mathrm{p}=0.002\right.$; fig. $\left.2 \mathrm{~b}\right)$ and no main effect of sex. Although the effect of breeding status on regional volume appeared larger for males in the BSTp and larger for females in the PVN, neither sex-by-status interaction term reached significance $\left(\mathrm{F}_{1,16}=3.48 ; \mathrm{p}=\right.$ 0.08 for the BSTp and $\mathrm{F}_{1,16}=3.99 ; \mathrm{p}=0.06$ for the PVN).

In contrast to the BSTp and PVN, MeA volume did not differ according to status and there was no sex-by-status interaction. Instead, a significant main effect of sex was detected $\left(\mathrm{F}_{1,16}=5.79 ; \mathrm{p}=0.03\right.$; fig. $\left.2 \mathrm{c}\right)$, with greater MeA volumes in males. There was no effect of sex or status and no sex-by-status interaction for SCN volume (all $\mathrm{p}$ values $>0.2$; fig. $2 \mathrm{~d}$ ).

Cell size differed by breeding status in the PVN $\left(\mathrm{F}_{1,16}=9.12 ; \mathrm{p}=0.008\right.$; fig. $\left.3 \mathrm{~b}\right)$ and $\mathrm{MeA}\left(\mathrm{F}_{1,16}=8.79 ; \mathrm{p}=\right.$ 0.01 ; fig. $3 c$ ); in both cases, breeders had larger cells than did subordinates. No main effects of sex and no sex-bystatus interactions on cell size were detected in any brain region.

In regression analyses, no statistically significant relationships between age and brain morphology were detected, with the single exception of cell size in the SCN 
Fig. 2. Regional volume (mean + SEM) for the BSTp (a), PVN (b), MeA (c), and SCN (d) of subordinate and breeder Damaraland mole-rats. The number of animals per group is noted at the base of each bar. ${ }^{\text {a Sig- }}$ nificant main effect of social status; ${ }^{b}$ significant main effect of sex.

Fig. 3. Cell size (mean + SEM) for the BSTp (a), PVN (b), MeA (c), and SCN (d) of subordinate and breeder Damaraland molerats. The number of animals per group is noted at the base of each bar. ${ }^{\text {a }}$ Significant main effect of social status.
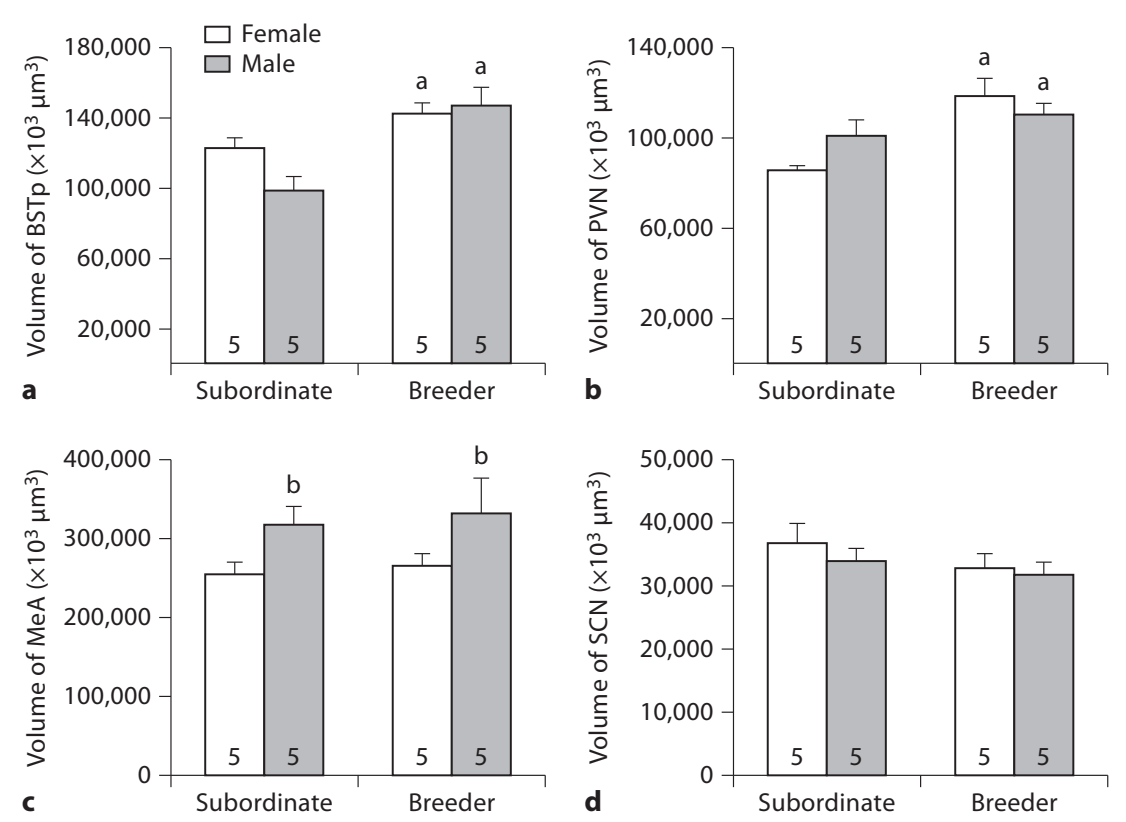

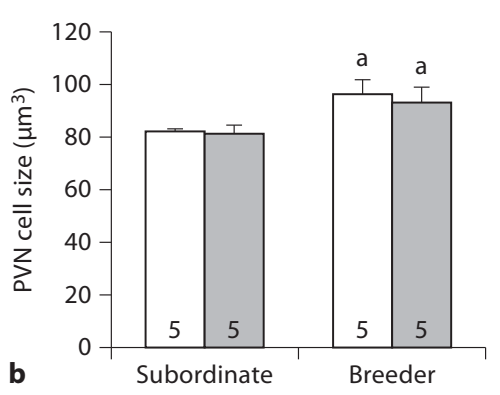

a
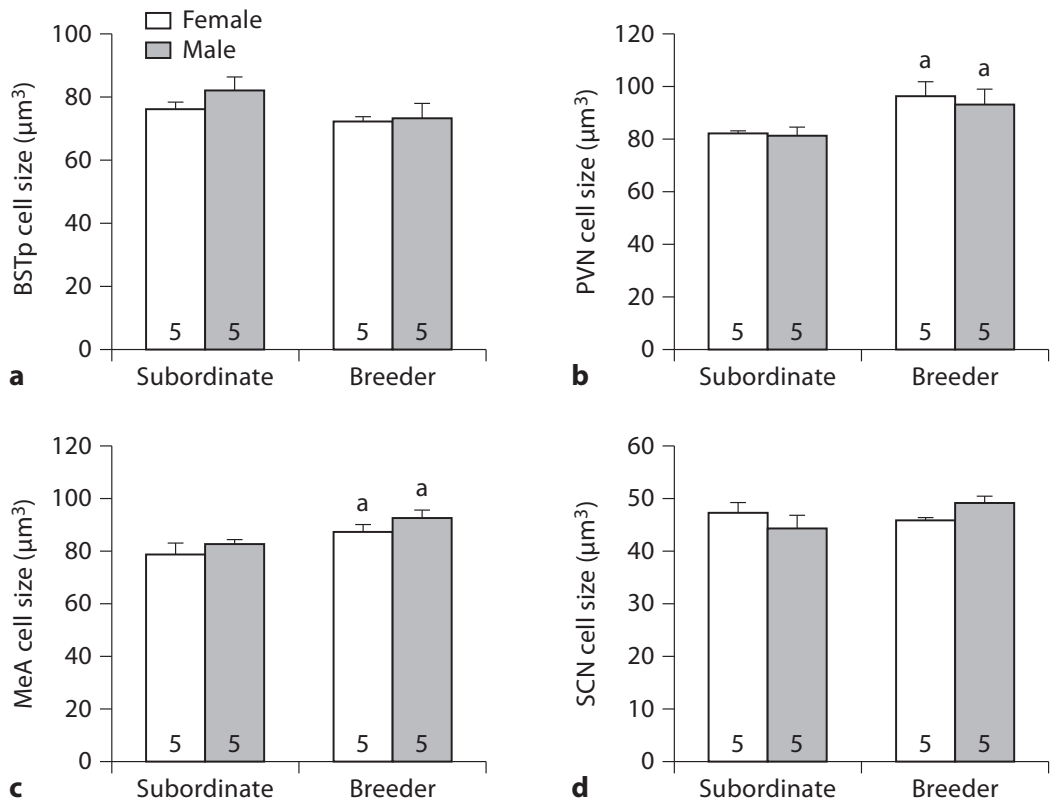

being positively correlated with age in subordinates only (table 2).

\section{Spinal Motoneurons}

Males had significantly more $\mathrm{ON}$ motoneurons than did females $\left(\mathrm{F}_{1,16}=8.62 ; \mathrm{p}=0.01\right.$; fig. $\left.4 \mathrm{a}\right)$. There was no significant effect of status, but the sex-by-status interaction approached significance $\left(F_{1,16}=3.912 ; p=0.07\right)$, with the effect of status being larger in males. Neither sex nor status affected $\mathrm{ON}$ motoneuron soma size although a significant interaction was detected $\left(\mathrm{F}_{1,16}=5.84 ; \mathrm{p}=0.03\right.$; fig. $4 \mathrm{~b})$. No significant effects of sex or status and no sex- 
Fig. 4. Cell number (a) and cell size (b) $($ mean + SEM) for ON of subordinate and breeder Damaraland mole-rats. The number of animals per group is noted at the base of each bar. ${ }^{\mathrm{b}}$ Significant main effect of sex.

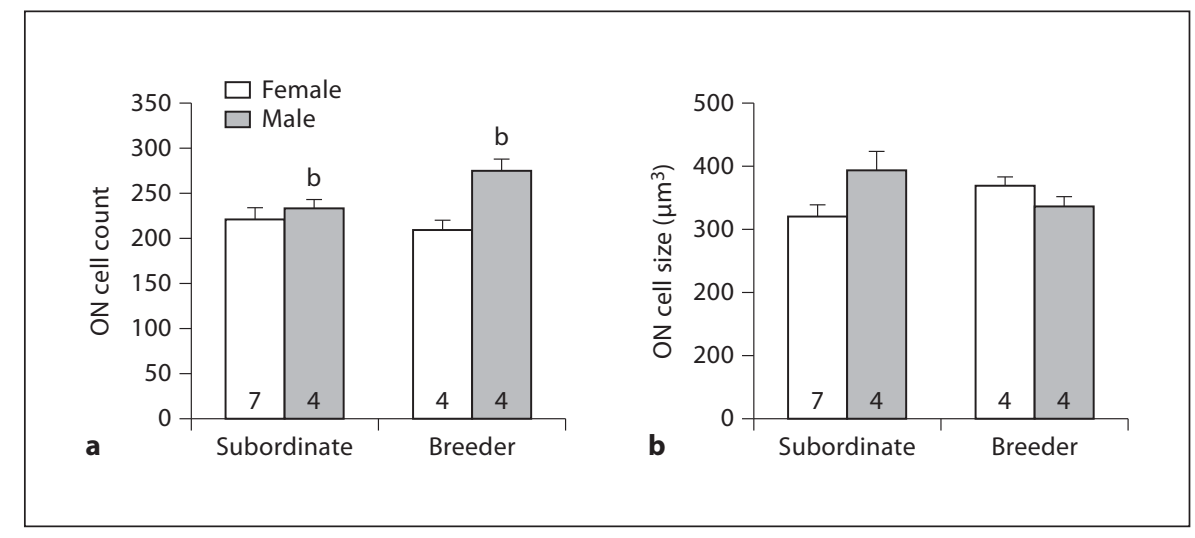

Table 2. Results of statistical regression analyses between age and all neural measurements reported for breeders and subordinates (males and females combined)

\begin{tabular}{llll}
\hline & & Breeders & Subordinates \\
\hline BSTp & $\begin{array}{l}\text { volume } \\
\text { cell size }\end{array}$ & $\mathrm{R}^{2}=0.16 ; \mathrm{p}=0.25$ & $\mathrm{R}^{2}=0.01 ; \mathrm{p}=0.85$ \\
PVN & volume & $\mathrm{R}^{2}=0.08 ; \mathrm{p}=0.43$ & $\mathrm{R}^{2}=0.19 ; \mathrm{p}=0.21$ \\
& cell size & $\mathrm{R}^{2}=0.11 ; \mathrm{p}=0.34$ & $\mathrm{R}^{2}=0.37 ; \mathrm{p}=0.06$ \\
MeA & volume & $\mathrm{R}^{2}=0.01 ; \mathrm{p}=0.83$ & $\mathrm{R}^{2}=0.03 ; \mathrm{p}=0.65$ \\
& cell size & $\mathrm{R}^{2}=0.09 ; \mathrm{p}=0.40$ & $\mathrm{R}^{2}=0.16 ; \mathrm{p}=0.25$ \\
SCN & volume & $\mathrm{R}^{2}=0.22 ; \mathrm{p}=0.17$ & $\mathrm{R}^{2}=0.10 ; \mathrm{p}=0.38$ \\
& cell size & $\mathrm{R}^{2}=0.09 ; \mathrm{p}=0.39$ & $\mathrm{R}^{2}=0.57 ; \mathrm{p}=0.01^{*}$ \\
ON & cell number & $\mathrm{R}^{2}=0.11 ; \mathrm{p}=0.47$ & $\mathrm{R}^{2}=0.02 ; \mathrm{p}=0.72$ \\
& cell size & $\mathrm{R}^{2}=0.03 ; \mathrm{p}=0.69$ & $\mathrm{R}^{2}=0.04 ; \mathrm{p}=0.58$ \\
\hline
\end{tabular}

* Statistically significant relationship between age and morphological measurement.

by-status interactions were detected for either RDLN motoneuron number or cell size (not shown).

No statistically significant relationships were detected between age and ON motoneuron number or cell size (table 2).

\section{Discussion}

We previously found no sexual dimorphism in any of the several brain regions examined in naked mole-rats, but breeders, regardless of sex, had larger volumes of the BSTp, PVN, and MeA than did subordinates [Holmes et al., 2007]. Similarly, the present data indicate that reproductive status influences regional brain volume in Dama- raland mole-rats: both the BSTp and PVN were larger in breeders than in subordinates. The effect of status also extended to cell size in some regions; breeders had larger cells in the PVN and MeA than did subordinates. In contrast to what was seen previously for naked mole-rats, however, we also observed sexual dimorphisms in Damaraland mole-rats. Males had larger MeA volumes than did females, regardless of status. A similar pattern was seen in the number of motoneurons in ON (i.e. males had more motoneurons than did females) although in this case the effect was clearly larger among breeders than subordinates. Collectively, these data reveal that both social status and sex influence the morphology of reproductive neural regions in this species and appear to do so in a region-specific manner.

Because breeders were older than subordinates in this study, we cannot rule out the possibility that differences between the subordinate and breeder groups were due to age and not social status. This seems unlikely for several reasons. First, all of the Damaraland mole-rats in the current study were well into adulthood, yet far from senescence, and breeders and subordinates did not differ in body size. We also do not know of any example of progressive increases in nuclear volume with age among adult animals (although there are some examples of shrinking with aging). In regression analyses, while we did see a significant relationship between age and SCN cell size in subordinates (table 2), no other relationships between age and neural morphology were detected. Similarly, there were no significant correlations between age and neural morphology in naked mole-rats [Holmes et al., 2011]. Therefore, it seems more likely that the changes in both species of mole-rat reflect differences in reproductive and social status. 
The function of the changes seen in breeders is not known. The neural changes may be required to trigger behavioral and neuroendocrine functioning associated with reproduction or may be a response to changes in the hormonal or social milieu that accompany the transition to breeding status. The PVN is a crucial node at the crossroads of the hypothalamic-pituitary-adrenal and hypothalamic-pituitary-gonadal axes. The BSTp and MeA are involved in the processing of pheromonal input in other rodents and have associated roles in controlling neuroendocrine function and sexual behavior [Simerly, 2002]. The BSTp has also been implicated in the regulation of stress responses in other rodents via its projections to the PVN [Choi et al., 2008]. While the relationship between stress and social status in naked mole-rats is not simple [Clarke and Faulkes, 1997, 1998], it seems likely that at least some components of the hypothalamic-pituitaryadrenal axis are affected when an animal becomes a breeder. Furthermore, most sex differences in other species are due to either developmental or adult differences in gonadal hormones [Morris et al., 2004]. Presumably gonadal hormones affect in some way the structure and/ or function of reproductive brain nuclei in the mole-rat brain, although we have yet to demonstrate this.

We did not see an effect of breeding status on MeA volume in Damaraland mole-rats. We previously reported a breeding status effect in naked mole-rats when we compared subordinates to gonadally intact breeders living within their colony [Holmes et al., 2007]. More recently, however, we saw no effect of status on MeA volume comparing subordinate naked mole-rats to breeders that had produced pups but not raised them (i.e. animals were paired but because no pups were raised they were not in a colony) [Holmes et al., 2011]. Similarly, the breeding Damaraland mole-rats in the present report were paired animals that had produced at least 1 litter but had not successfully raised any pups to adulthood. Thus, status effects on MeA morphology may require the breeding animals to be housed within a colony (i.e. to actually be socially dominant). This is an intriguing idea that warrants further investigation. Regardless, the effect of sex on MeA morphology that we report distinguishes the Damaraland mole-rat from both of our previous naked molerat reports [Holmes et al., 2007, 2011]. We also found a sex difference in $\mathrm{ON}$ motoneuron number in Damaraland mole-rats consistent with all other mammalian species studied [e.g. Breedlove and Arnold, 1980; Forger and Breedlove, 1986, 1987; Ulibarri et al., 1995; Polak and Freeman, 2010], with the exception of the naked mole-rat [Peroulakis et al., 2002; Seney et al., 2006]. Thus, the cur- rent findings demonstrate that sex can influence neural morphology in a eusocial mammal.

We propose that differences in the degree of sexual dimorphism between naked mole-rats and Damaraland mole-rats are related to species differences in reproductive skew. Damaraland mole-rats live in smaller colonies and have smaller litters; as a result, subordinates have a greater likelihood of becoming reproductive relative to naked mole-rats [Jarvis et al., 1994; Bennett and Faulkes, 2000]. Thus, Damaraland mole-rats may be somewhat of an intermediate species in terms of reproductive skew: they show more reproductive skew than laboratory rats or mice but less than naked mole-rats. Indeed, we have recently demonstrated that the morphology of the external genitalia of these animals is related to social structure [Seney et al., 2009]. Specifically, we compared the genitals and perineal muscles of Damaraland mole-rats, naked mole-rats, and silvery mole-rats (Heliophobius argenteocinereus), a solitary mole-rat species in which all individuals that reach adulthood may reproduce [Bennett and Faulkes, 2000]. We found that naked mole-rats lacked sex differences in genitalia and perineal morphology (and, indeed, had status differences), silvery mole-rats exhibited sex differences in these measures, and Damaraland mole-rats were intermediate [Seney et al., 2009].

African mole-rats exhibit a vast range of sociality within a phylogenetically similar group, thus providing the opportunity to test hypotheses regarding evolved associations between social structure, reproductive skew and physiology, anatomy, and behavior. The present data demonstrate that social status influences neural morphology in Damaraland mole-rats, as it does in the eusocial naked mole-rat, but also reveals sex differences in the Damaraland mole-rat nervous system. This species appears intermediate between the highly social naked molerat and less social traditional laboratory rodents when it comes to the degree of sexual differentiation of the nervous system. We propose that the relative importance of social status and sex in organizing neural morphology is the result of species differences in reproductive skew and, proximately, is likely due to species-specific specializations in neuroendocrine function.

\section{Acknowledgements}

The authors are grateful to Sharry Goldman for invaluable assistance with animal husbandry. This work was supported by the NSF (IOS-0642050 to N.G.F. and B.D.G.), the NIMH (K02 MH072825 to N.G.F.), the NINDS (F31 NS058258 to M.L.S.), and the NSERC (Discovery Grant to M.M.H.). 


\section{References}

-Allard MW, Honeycutt RL (1992): Nucleotide sequence variation in the mitochondrial $12 \mathrm{~S}$ rRNA gene and the phylogeny of African mole-rats (Rodentia: Bathyergidae). Mol Biol Evol 9:27-40.

Bennett NC, Faulkes CG (2000): African MoleRats: Ecology and Eusociality. Cambridge, Cambridge University Press.

Bennett NC, Jarvis JUM (1988): The social structure and reproductive biology of colonies of the mole-rat Cryptomys damarensis (Rodentia: Bathyergidae). J Mammal 69:293-302.

- Bennett NC, Jarvis JUM, Aguilar GH, McDaid EJ (1991): Growth and development in six species of African mole-rats (Rodentia: Bathyergidae). J Zool 225:13-26.

Breedlove SM, Arnold AP (1980): Hormone accumulation in a sexually dimorphic motor nucleus of the rat spinal cord. Science 210: 564-566.

Choi DC, Furay AR, Evanson NK, Ulrich-Lai YM, Nguyen MM, Ostrander MM, Herman JP (2008): The role of the posterior medial bed nucleus of the stria terminalis in modulating hypothalamic-pituitary-adrenocortical axis responsiveness to acute and chronic stress. Psychoneuroendocrinology 33:659669.

Clarke FM, Faulkes CG (1997): Dominance and queen succession in captive colonies of the eusocial naked mole-rat, Heterocephalus glaber. Proc Biol Sci 264:993-1000.

Clarke FM, Faulkes CG (1998): Hormonal and behavioural correlates of male dominance and reproductive status in captive colonies of the naked mole-rat, Heterocephalus glaber. Proc Biol Sci 265:1391-1399.

Forger NG, Breedlove SM (1986): Sexual dimorphism in human and canine spinal cord: role of early androgen. Proc Natl Acad Sci USA 83:7527-7531.
Forger NG, Breedlove SM (1987): Motoneuronal death during human fetal development. J Comp Neurol 264:118-122.

Franklin KB, Paxinos G (2008): The Mouse Brain in Stereotaxic Co-Ordinates, ed 3. New York, Academic Press.

Gorski RA, Gordon JH, Shryne JE, Southam AM (1978): Evidence for a morphological sex difference within the medial preoptic area of the rat brain. Brain Res 148:333-346.

Holmes MM, Rosen GJ, Jordan CL, De Vries GJ, Goldman BD, Forger NG (2007): Social control of brain morphology in a eusocial mammal. Proc Natl Acad Sci USA 104:1054810552.

-Holmes MM, Seney ML, Goldman BD, Forger NG (2011): Social and hormonal triggers of neural plasticity in naked mole-rats. Behav Brain Res 218:234-239.

-Jarvis JUM (1981): Eusociality in a mammal cooperative breeding in naked mole-rat Heterocephalus glaber colonies. Science 212: 571-573.

Jarvis JUM (1991): Reproduction of naked molerats; in Sherman PW, Jarvis JUM, Alexander RD (eds): Biology of the Naked Mole-Rat. New Jersey, Princeton University Press, pp 384-425.

-Jarvis JUM, Bennett NC (1993): Eusociality has evolved independently in two genera of Bathyergid mole-rats - but occurs in no other subterranean mammal. Behav Ecol Sociobiol 33:253-260.

Jarvis JUM, O'Riain MJ, Bennett NC, Sherman PW (1994): Mammalian eusociality: a family affair. Trends Ecol Evol 9:47-51.

Kock D, Ingram CM, Frabotta LJ, Honeycutt RL, Burda H (2006): On the nomenclature of Bathyergidae and Fukomys n. gen. (Mammalia: Rodentia). Zootaxa 1142:51-55.

Lacey EA, Alexander RD, Braude SH, Sherman PW, Jarvis JUM (1991): An ethogram for the naked mole-rat: nonvocal behaviors; in Sherman PW, Jarvis JU, Alexander RD (eds): Biology of the Naked Mole-Rat. New Jersey, Princeton University Press, pp 209-242.
Lacey EA, Sherman PW (1991): Social organization of naked mole-rat colonies: evidence for divisions of labor; in Sherman PW, Jarvis JUM, Alexander RD (eds): Biology of the Naked Mole-Rat. New Jersey, Princeton University Press, pp 275-336.

Morris JA, Jordan CL, Breedlove SM (2004): Sexual differentiation of the vertebrate nervous system. Nat Neurosci 7:1034-1039.

-Peroulakis ME, Goldman BD, Forger NG (2002): Perineal muscles and motoneurons are sexually monomorphic in the naked mole-rat (Heterocephalus glaber). J Neurobiol 51:3342.

Polak K, Freeman LM (2010): Sex difference in Onuf's nucleus homologue in the Asian musk shrew. Brain Res 1346:62-68.

- Seney M, Goldman BD, Forger NG (2006): Breeding status affects motoneuron number and muscle size in naked mole-rats: recruitment of perineal motoneurons? J Neurobiol 66:1354-1364.

-Seney ML, Kelly DA, Goldman BD, Sumbera R, Forger NG (2009): Social structure predicts genital morphology in African mole-rats. PLoS One 4:e7477.

- Simerly RB (2002): Wired for reproduction: organization and development of sexually dimorphic circuits in the mammalian forebrain. Annu Rev Neurosci 25:507-536.

Sura R, French RA, Goldman BD, Schwartz DR (2010): Neoplasia and granulomas surrounding microchip transponders in Damaraland mole rats (Cryptomys damarensis). Vet Pathol.

-Ulibarri C, Popper P, Micevych PE (1995): Motoneurons dorsolateral to the central canal innervate perineal muscles in the Mongolian gerbil. J Comp Neurol 356:225-237. 